\title{
Is model-order reduction viable for the broadband finite-element analysis of electrically large antenna arrays?
}

\author{
O. Floch, A. Sommer, O. Farle, and R. Dyczij-Edlinger \\ Chair of Electromagnetic Theory, Dept. of Physics and Mechatronics, Saarland University, Saarbrücken, Germany \\ Correspondence to: O. Floch (o.floch@1te.uni-saarland.de)
}

Received: 26 January 2015 - Accepted: 24 March 2015 - Published: 3 November 2015

\begin{abstract}
Model-order reduction provides an efficient way of computing frequency sweeps for finite-element models, because the dimension of the reduced-order system depends on the complexity of the frequency response rather than the size of the original model. For electrically large domains, however, the applicability of such methods is unclear because the system response may be very complicated. This paper provides a numerical study of the effects of bandwidth, electrical size, and scan angle on the size and convergence of the ROM, by considering linear antenna arrays. A mathematical model is proposed and validated against numerical experiments.
\end{abstract}

\section{Introduction}

Numerical volume discretization methods, such as finiteelements (FE), provide a highly flexible approach to the solution of electromagnetic boundary value problems. The resulting systems of linear equations are typically sparse but of very large scale. They are commonly solved by direct methods, due to their high robustness. However, both computational complexity and memory requirements of such methods are sub-optimal. Iterative solvers, like Krylov sub-space techniques, provide an attractive alternative which, however, depends critically on the availability of efficient preconditioners.

In the frequency domain, the structure of the underlying indefinite vector Helmholtz equation and the effect of dispersion error have long impeded the development of effective preconditions for domains that are electrically large. Recent advances in domain decomposition (DD), as reported in Lee et al. (2005) and Rawat and Lee (2007), have greatly facilitated the solution of the resulting FE systems, especially in case of antenna arrays. Nevertheless, such methods just provide solutions at single frequency points.

When broad frequency bands are to be analyzed, multipoint methods of model-order reduction (MOR) are more attractive than conventional FE analysis. These techniques employ well-chosen snapshots of the solution manifold to generate an approximation space whose dimension is low and does not depend on the size of the underlying system. Stateof-the-art MOR approaches place expansion points adaptively, based on some a posteriori error indicator Chen et al. (2010), Hesthaven et al. (2012).

The dimension of the reduced-order system (ROM) does, however, depend on the complexity of the system response for the specific excitations under consideration. Therefore, it is unclear whether MOR is suitable for the FE-based broadband analysis of large-scale antenna arrays. On the one hand, these structures are electrically large and exhibit high numbers of independent inputs. Moreover, the individual radiators often exhibit resonant behavior. Thus, the resulting frequency response may be expected to be complicated and require a large ROM to be represented well. On the other hand, the considered structures serve the prime purpose of radiating electromagnetic waves. Thus, even though the array may support a great number of beam forms and a wide range of scan angles, the excitations and fields of the individual elements are always characterized by a large amount of phase coherence and slowly changing amplitude distributions. Such system properties are well-suited for MOR.

The first goal of this paper is to numerically investigate the effects of bandwidth, electrical size, and scan angle on the dimension and convergence properties of the ROM. For this purpose, we consider linear arrays of up to 144 wavelengths $\lambda$ in size. We restrict ourselves to these essentially onedimensional structures, because they allow to study large- 
scale effects at minimum computational costs. Nevertheless, for the largest structure the number of FE unknowns exceeds 22 million. Therefore, all FE computations are performed by a general-purpose DD solver which has successfully been applied to truly three-dimensional structures; see Rawat and Lee (2010). Based on the numerical results, we propose a simple mathematical model based on sampling theory to predict the convergence properties of the ROM. The model is validated by comparing its predictions against the results of additional numerical experiments. In view of the general principles behind their core findings, the authors conjecture that they will transfer well to planar and general array configurations.

\section{Problem formulation}

\subsection{Time-harmonic boundary value problem}

The electric field is denoted by $\boldsymbol{E}$, the magnetic field by $\boldsymbol{H}$, the surface current density by $\boldsymbol{K}$, the relative magnetic permeability by $\mu_{\mathrm{r}}$, and the relative electric permittivity by $\epsilon_{\mathrm{r}}$. The abbreviations $k_{0}$ and $\eta_{0}$ stand for the free-space wave number and characteristic impedance, respectively.

Given a bounded domain $\Omega \subset \mathbf{R}^{3}$ with boundary $\partial \Omega=$ $\Gamma_{P} \cup \Gamma_{E} \cup \Gamma_{A}$ and unit outward normal vector $\hat{\boldsymbol{n}}$, we consider the time-harmonic boundary value problem (BVP)

$$
\begin{aligned}
\nabla \times \mu_{r}^{-1} \nabla \times \boldsymbol{E}-k_{0}^{2} \epsilon_{r} \boldsymbol{E} & =\mathbf{0} \quad \text { in } \Omega, \\
\pi_{t}(\boldsymbol{E}) & =\mathbf{0}, \quad \text { on } \Gamma_{E}, \\
{\left[\mu_{r}^{-1} \nabla \times \boldsymbol{E}\right] } & =-j k_{0} \eta_{0} \boldsymbol{K}, \text { on } \Gamma_{P}, \\
\gamma_{t}\left(\mu_{\mathrm{r}}^{-1} \nabla \times \boldsymbol{E}\right)-j k_{0} \sqrt{\frac{\epsilon_{\mathrm{r}}}{\mu_{\mathrm{r}}}} \pi_{t}(\boldsymbol{E}) & =\mathbf{0}, \quad \text { on } \Gamma_{A} .
\end{aligned}
$$

Here, $\gamma_{t}(\boldsymbol{E})=\boldsymbol{n} \times \boldsymbol{E}$ denotes the tangential trace and $\pi_{t}(\boldsymbol{E})=\hat{\boldsymbol{n}} \times(\boldsymbol{E} \times \hat{\boldsymbol{n}})$ the tangential component trace mapping; see Buffa and Ciarlet (2001). The jump operator $[\boldsymbol{E}]$ is defined by

$$
[\boldsymbol{E}]:[\boldsymbol{E}]_{i j}=\gamma_{t}\left(\boldsymbol{E}_{i}\right)+\gamma_{t}\left(\boldsymbol{E}_{j}\right) .
$$

For clarity of presentation, we only consider two subdomains in the DD formulation and assume that a single material region is cut by the interface $\Gamma$ : We decompose $\Omega$ into two non-overlapping sub-domains such that $\bar{\Omega}=\overline{\Omega_{1} \cup \Omega_{2}}$, $\Omega_{1} \cap \Omega_{2}=\varnothing, \Gamma=\overline{\Omega_{1}} \cap \overline{\Omega_{2}}$, and denote the interface seen from $\Omega_{1}$ and $\Omega_{2}$, respectively, by $\Gamma_{12}$ and $\Gamma_{21}$. For the boundaries of the sub-domains, we use the notation $\Gamma_{X}^{m}=$ $\partial \Omega_{m} \cap \Gamma_{X}$ with $X \in\{E, P, A\}$ and $m \in\{1,2\}$. The decom- posed BVP reads

$$
\begin{aligned}
& \nabla \times \mu_{\mathrm{r}, 1}^{-1} \nabla \times \boldsymbol{E}_{1}-k_{0}^{2} \epsilon_{\mathrm{r}, 1} \boldsymbol{E}_{1}=\mathbf{0} \quad \text { in } \Omega_{1}, \\
& \nabla \times \mu_{\mathrm{r}, 2}^{-1} \nabla \times \boldsymbol{E}_{2}-k_{0}^{2} \epsilon_{\mathrm{r}, 2} \boldsymbol{E}_{2}=\mathbf{0} \text { in } \Omega_{2}, \\
& \pi_{t}\left(\boldsymbol{E}_{1}\right)=\mathbf{0} \text { on } \Gamma_{E}^{1}, \\
& \pi_{t}\left(\boldsymbol{E}_{2}\right)=\mathbf{0} \text { on } \Gamma_{E}^{2}, \\
& {\left[\mu_{\mathrm{r}, 1}^{-1} \nabla \times \boldsymbol{E}_{1}\right]=-j k_{0} \eta_{0} \boldsymbol{K}_{1},} \\
& \text { on } \Gamma_{P}^{1} \\
& {\left[\mu_{\mathrm{r}, 2}^{-1} \nabla \times \boldsymbol{E}_{2}\right]=-j k_{0} \eta_{0} \boldsymbol{K}_{2},} \\
& \text { on } \Gamma_{P}^{2} \\
& \gamma_{t}\left(\mu_{\mathrm{r}, 1}^{-1} \nabla \times \boldsymbol{E}_{1}\right)-j k_{0} \sqrt{\frac{\epsilon_{\mathrm{r}, 1}}{\mu_{\mathrm{r}, 1}}} \pi_{t}\left(\boldsymbol{E}_{1}\right)=\mathbf{0} \quad \text { on } \Gamma_{A}^{1}, \\
& \gamma_{t}\left(\mu_{\mathrm{r}, 2}^{-1} \nabla \times \boldsymbol{E}_{2}\right)-j k_{0} \sqrt{\frac{\epsilon_{\mathrm{r}, 2}}{\mu_{\mathrm{r}, 2}}} \pi_{t}\left(\boldsymbol{E}_{2}\right)=\mathbf{0} \quad \text { on } \Gamma_{A}^{2}, \\
& \mathcal{T}\left(\boldsymbol{E}_{1}\right)-\mathcal{T}\left(\boldsymbol{E}_{2}\right)=\mathbf{0} \quad \text { on } \Gamma_{12}, \\
& \mathcal{T}\left(\boldsymbol{E}_{2}\right)-\mathcal{T}\left(\boldsymbol{E}_{1}\right)=\mathbf{0} \quad \text { on } \Gamma_{21} .
\end{aligned}
$$

Equations $3 \mathrm{i}$ and $3 \mathrm{j}$ enforce the necessary continuity requirements for the electric and magnetic fields on the interface. We here consider a transmission condition $\mathcal{T}$ with two transverse derivatives of second order,

$$
\begin{aligned}
& \mathcal{T}(\boldsymbol{E})=\alpha \pi_{t}(\boldsymbol{E})+\beta \nabla_{t} \times \nabla_{t} \times \pi_{t}(\boldsymbol{E}) \\
& +\gamma \nabla_{t} \nabla_{t} \cdot \gamma_{t}\left(\mu_{\mathrm{r}}^{-1} \nabla \times \boldsymbol{E}\right),
\end{aligned}
$$

with complex coefficients $\alpha, \beta$ and $\gamma$. It was shown in Rawat and Lee (2010) that equivalence of Eqs. (3) and (1) is guaranteed provided that $\Im\left(\frac{\beta}{\alpha}\right) \neq 0$ or $\Re\left(\frac{\beta}{\alpha}\right) \geq 0$ and $\Im(\gamma) \neq 0$ or $\Re(\gamma) \geq 0$. See Dolean et al. (2015) for a more detailed discussion.

\subsubsection{Finite-element formulation}

Let $\boldsymbol{H}^{\text {curl }}(\Omega), H^{1}(\Omega), \boldsymbol{H}^{\frac{1}{2}}(\Gamma)$, and $H^{\frac{1}{2}}(\Gamma)$ denote the Sobolev spaces from Buffa and Ciarlet (2001). We define the subspace $\boldsymbol{H}_{E}^{\text {curl }}$ by

$\boldsymbol{H}_{E}^{\text {curl }}(\Omega):=\left\{\boldsymbol{v} \in \boldsymbol{H}^{\text {curl }}(\Omega) \mid \pi_{t}(\boldsymbol{v})=0\right.$ on $\left.\Gamma_{E}\right\}$

and denote the topological dual spaces of $H^{\frac{1}{2}}\left(\Gamma_{m n}\right)$ and $\boldsymbol{H}^{\frac{1}{2}}\left(\Gamma_{m n}\right)$ by $H^{-\frac{1}{2}}\left(\Gamma_{m n}\right)$ and $\boldsymbol{H}^{-\frac{1}{2}}\left(\Gamma_{m n}\right)$, respectively. To implement the DD formulation 3 , we introduce auxiliary variables $\boldsymbol{j}_{m} \in \boldsymbol{H}^{-\frac{1}{2}}\left(\Gamma_{m n}\right)$ and $p_{m} \in H^{-\frac{1}{2}}\left(\Gamma_{m n}\right)$ on the interfaces, by

$$
\begin{array}{ll}
\boldsymbol{j}_{m}=k_{0}^{-1} \gamma_{t}\left(\mu_{\mathrm{r}, \mathrm{m}}^{-1} \nabla \times \boldsymbol{E}_{m}\right), & m \in\{1,2\}, \\
p_{m}=\left(k_{0}^{2}\right)^{-1} \nabla_{t} \cdot \gamma_{t}\left(\mu_{\mathrm{r}, \mathrm{m}}^{-1} \nabla \times \boldsymbol{E}_{m}\right), & m \in\{1,2\} .
\end{array}
$$

The corresponding FE spaces are denoted by $\mathcal{V}_{m, E}^{\text {curl }} \subset$ $\boldsymbol{H}_{E}^{\text {curl }}\left(\Omega_{m}\right), \mathcal{V}_{m n}^{\Gamma} \subset \boldsymbol{H}^{-\frac{1}{2}}\left(\Gamma_{m n}\right)$, and $\mathcal{V}_{m n}^{\Gamma} \subset H^{-\frac{1}{2}}\left(\Gamma_{m n}\right)$. The 
FE spaces for the auxiliary variables $\boldsymbol{j}_{m}$ and $p_{m}$ are taken to be the range of the tangential component map $\pi_{t}\left(\mathcal{V}_{m, E}^{\text {curl }}\right)$ and the two-dimensional grad-conforming space, respectively. The authors' computer implementation employs basis functions of second order. In the discretization process, we apply a Galerkin procedure to Eqs. (3j) and (3b), substitute Eq. (6) for the resulting surface terms, and test the interface condition as well as the definition Eq. (7) by shape functions $\boldsymbol{u} \in \mathcal{V}_{m n}^{\Gamma}$ and $\phi \in \mathcal{V}_{m n}^{\Gamma}$, respectively. The resulting FE-DD system reads

$$
\left[\begin{array}{cc}
\mathbf{A}_{1}\left(k_{0}, \xi\right) & \mathbf{C}_{12}\left(k_{0}, \xi\right) \\
\mathbf{C}_{21}\left(k_{0}, \xi\right) & \mathbf{A}_{2}\left(k_{0}, \xi\right)
\end{array}\right]\left[\begin{array}{l}
\boldsymbol{x}_{1}\left(k_{0}\right) \\
\boldsymbol{x}_{2}\left(k_{0}\right)
\end{array}\right]=j k_{0} \eta_{0}\left[\begin{array}{l}
\boldsymbol{b}_{1}\left(k_{0}\right) \\
\boldsymbol{b}_{2}\left(k_{0}\right)
\end{array}\right],
$$

wherein $\xi=(\alpha, \beta, \gamma), \quad \mathbf{A}_{m} \in \mathbf{C}^{N_{m} \times N_{m}}, \quad \mathbf{C}_{m n} \in \mathbf{C}^{N_{m} \times N_{n}}$, and $\quad \boldsymbol{b}_{m} \in \mathbf{C}^{N_{m}}$, with $m, n \in\{1,2\}$. The vectors $\boldsymbol{x}_{m}=\boldsymbol{e}_{m}, \boldsymbol{j}_{m}^{\Gamma}, \boldsymbol{p}_{m}^{\Gamma}{ }^{T}$ are of dimension $N_{m}$ and include FE degrees of freedom for both the electric field $\boldsymbol{E}_{m}$ and the auxiliary variables $\boldsymbol{j}_{m}$ and $\boldsymbol{p}_{m}$ inside the sub-domain $\Omega_{m}$. The corresponding block matrices are given by

$$
\begin{aligned}
\mathbf{A}_{m} & =\left[\begin{array}{ccc}
\mathbf{A}_{m}^{e, e}-k_{0}^{2} \mathbf{B}_{m}^{e, e}+k_{0} \mathbf{R}_{m}^{e, e} & k_{0} \mathbf{T}_{m}^{e, j} & \mathbf{0} \\
k_{0}\left(\mathbf{T}_{m}^{e, j}\right)^{T}+\frac{k_{0} \beta}{\alpha} \mathbf{S}_{m}^{j, e} & \frac{k_{0}^{2}}{\alpha} \mathbf{T}_{m}^{j, j} & \frac{k_{0}^{3} \gamma}{\alpha} \mathbf{D}_{m}^{j, p} \\
\mathbf{0} & \mathbf{F}_{m}^{p, j} & k_{0} \mathbf{T}_{m}^{p, p}
\end{array}\right], \\
\mathbf{C}_{m n} & =\left[\begin{array}{ccc}
\mathbf{0} & \mathbf{0} & \mathbf{0} \\
-k_{0}\left(\mathbf{T}_{m n}^{e, j}\right)^{T}-\frac{k_{0} \beta}{\alpha} \mathbf{S}_{m n}^{j, e} & \frac{k_{0}^{2}}{\alpha} \mathbf{T}_{m n}^{j, j} & \frac{k_{0}^{3} \gamma}{\alpha} \mathbf{D}_{m n}^{j, p} \\
\mathbf{0} & \mathbf{0} & \mathbf{0}
\end{array}\right], \\
\boldsymbol{b}_{m} & =\left[\begin{array}{c}
\boldsymbol{y}_{m} \\
\mathbf{0} \\
\mathbf{0}
\end{array}\right]
\end{aligned}
$$

wherein

$$
\begin{aligned}
& {\left[\mathbf{A}_{m}^{\boldsymbol{e}, \boldsymbol{e}}\right]_{i j}=\left(\nabla \times \boldsymbol{v}_{i}, \mu_{\mathrm{r}, \mathrm{j}}^{-1} \nabla \times \boldsymbol{v}_{j}\right)_{\Omega_{m}},} \\
& {\left[\mathbf{B}_{m}^{\boldsymbol{e}, \boldsymbol{e}}\right]_{i j}=\left(\boldsymbol{v}_{i}, \epsilon_{r, j} \boldsymbol{v}_{j}\right)_{\Omega_{m}},} \\
& {\left[\mathbf{R}_{m}^{\boldsymbol{e}, \boldsymbol{j}}\right]_{i j}=j\left(\pi_{t}\left(\boldsymbol{v}_{i}\right), \pi_{t}\left(\boldsymbol{v}_{j}\right)\right\rangle_{\Gamma_{A}^{m}},} \\
& {\left[\mathbf{T}_{m n}^{\boldsymbol{e}, j}\right]_{i j}=\left\langle\pi_{t}\left(\boldsymbol{v}_{i}\right), \boldsymbol{u}_{j}\right\rangle_{\Gamma^{m n}},} \\
& {\left[\mathbf{T}_{m n}^{j, j}\right]_{i j}=\left\langle\boldsymbol{u}_{i}, \boldsymbol{u}_{j}\right\rangle_{\Gamma^{m n}},} \\
& {\left[\mathbf{S}_{m n}^{\boldsymbol{j}, \boldsymbol{e}}\right]_{i j}=\left\langle\nabla \times \boldsymbol{u}_{i}, \nabla \times \pi_{t}\left(\boldsymbol{v}_{j}\right)\right\rangle_{\Gamma^{m n}},} \\
& {\left[\mathbf{D}_{m m}^{j, \boldsymbol{p}}\right]_{i j}=\left\langle\boldsymbol{u}_{i}, \nabla \phi_{j}\right\rangle_{\Gamma^{m n}},} \\
& {\left[\mathbf{T}_{m m}^{\boldsymbol{p}, \boldsymbol{p}}\right]_{i j}=\left\langle\phi_{i}, \phi_{j}\right\rangle_{\Gamma^{m n}},} \\
& {\left[\mathbf{F}_{m m}^{\boldsymbol{p}, \boldsymbol{j}}\right]_{i j}=\left\langle\nabla \phi_{i}, \boldsymbol{u}_{j}\right\rangle_{\Gamma^{m n}},} \\
& {\left[\boldsymbol{y}_{m}\right]_{i}=\left\langle\pi_{t}\left(\boldsymbol{v}_{i}\right), \gamma_{t}(\boldsymbol{H})\right\rangle_{\Gamma^{H}},}
\end{aligned}
$$

with $\boldsymbol{v}_{i}, \boldsymbol{v}_{j} \in \mathcal{V}_{m, E}^{\mathrm{curl}}$, and $\boldsymbol{u}_{i}, \boldsymbol{u}_{j} \in \mathcal{V}_{m n}^{\Gamma}$ and $\phi_{i}, \phi_{j} \in \mathcal{V}_{m n}^{\Gamma}$. The volume $(\ldots)$ and surface $\langle\ldots\rangle$ bilinear forms are given by

$$
\begin{aligned}
(\boldsymbol{u}, \boldsymbol{v})_{\Omega} & =\int_{\Omega} \boldsymbol{u} \cdot \boldsymbol{v} \mathrm{d} \Omega, \\
\langle\boldsymbol{u}, \boldsymbol{v}\rangle_{\Gamma} & =\int_{\Gamma} \boldsymbol{u} \cdot \boldsymbol{v} \mathrm{d} \Gamma, \\
\langle\phi, \psi\rangle_{\Gamma} & =\int_{\Gamma} \phi \psi \mathrm{d} \Gamma .
\end{aligned}
$$

\section{Reduced-order model}

As a prerequisite for projection-based MOR, the system Eq. 8 has to be rewritten in affinely decomposed form with respect to the wavenumber $k_{0}$. This is achieved by defining the following wavenumber-independent matrices:

$$
\begin{aligned}
& \mathbf{A}_{m}^{0}=\left[\begin{array}{ccc}
\mathbf{A}_{m}^{e, e} & \mathbf{0} & \mathbf{0} \\
\mathbf{0} & \mathbf{0} & \mathbf{0} \\
\mathbf{0} & \mathbf{F}_{m}^{p, j} & \mathbf{0}
\end{array}\right], \quad \mathbf{A}_{m}^{1}=\left[\begin{array}{ccc}
\mathbf{R}_{m}^{e, e} & \mathbf{T}_{m}^{e, j} & \mathbf{0} \\
\left(\mathbf{T}_{m}^{e, j}\right)^{T} & \mathbf{0} & \mathbf{0} \\
\mathbf{0} & \mathbf{0} & \mathbf{T}_{m}^{p, p}
\end{array}\right], \\
& \mathbf{A}_{m}^{2}=\left[\begin{array}{ccc}
-\mathbf{B}_{m}^{e, e} & \mathbf{0} & \mathbf{0} \\
\mathbf{0} & \mathbf{0} & \mathbf{0} \\
\mathbf{0} & \mathbf{0} & \mathbf{0}
\end{array}\right], \quad \mathbf{A}_{m}^{3}=\left[\begin{array}{ccc}
\mathbf{0} & \mathbf{0} & \mathbf{0} \\
\mathbf{S}_{m}^{j, \boldsymbol{e}} & \mathbf{0} & \mathbf{0} \\
\mathbf{0} & \mathbf{0} & \mathbf{0}
\end{array}\right], \\
& \mathbf{A}_{m}^{4}=\left[\begin{array}{ccc}
\mathbf{0} & \mathbf{0} & \mathbf{0} \\
\mathbf{0} & \mathbf{T}_{m}^{j, j} & \mathbf{0} \\
\mathbf{0} & \mathbf{0} & \mathbf{0}
\end{array}\right], \quad \mathbf{A}_{m}^{5}=\left[\begin{array}{ccc}
\mathbf{0} & \mathbf{0} & \mathbf{0} \\
\mathbf{0} & \mathbf{0} & \mathbf{D}_{m}^{j, p} \\
\mathbf{0} & \mathbf{0} & \mathbf{0}
\end{array}\right]
\end{aligned}
$$


and

$$
\begin{array}{rlrl}
\mathbf{C}_{m n}^{0}=\mathbf{0}, & \mathbf{C}_{m n}^{1}= & {\left[\begin{array}{ccc}
\mathbf{0} & \mathbf{0} & \mathbf{0} \\
-\left(\mathbf{T}_{m n}^{e, j}\right)^{T} & \mathbf{0} & \mathbf{0} \\
\mathbf{0} & \mathbf{0} & \mathbf{0}
\end{array}\right],} \\
\mathbf{C}_{m n}^{2}=\mathbf{0}, & & \mathbf{C}_{m n}^{3}=\left[\begin{array}{ccc}
\mathbf{0} & \mathbf{0} & \mathbf{0} \\
-\mathbf{S}_{m n}^{j, e} & \mathbf{0} & \mathbf{0} \\
\mathbf{0} & \mathbf{0} & \mathbf{0}
\end{array}\right], \\
\mathbf{C}_{m n}^{4}=\left[\begin{array}{ccc}
\mathbf{0} & \mathbf{0} & \mathbf{0} \\
\mathbf{0} & \mathbf{T}_{m n}^{j, j} & \mathbf{0} \\
\mathbf{0} & \mathbf{0} & \mathbf{0}
\end{array}\right], & \mathbf{C}_{m n}^{5}=\left[\begin{array}{ccc}
\mathbf{0} & \mathbf{0} & \mathbf{0} \\
\mathbf{0} & \mathbf{0} & \mathbf{D}_{m n}^{j, p} \\
\mathbf{0} & \mathbf{0} & \mathbf{0}
\end{array}\right],
\end{array}
$$

with $m, n \in\{1,2\}$. Thus 8 takes the form

$$
\begin{array}{r}
\left(\sum_{i=1}^{I} \phi_{i}\left(k_{0}\right) \hat{\mathbf{A}}_{i}\right) \hat{\boldsymbol{x}}\left(k_{0}\right)=\left(\sum_{j=1}^{J} \theta_{j}\left(k_{0}\right) \hat{\boldsymbol{b}}_{j}\right), \\
\boldsymbol{y}\left(k_{0}\right)=\left(\sum_{j=1}^{J} \eta_{j}\left(k_{0}\right) \hat{\boldsymbol{b}}_{j}^{T}\right) \hat{\boldsymbol{x}}\left(k_{0}\right),
\end{array}
$$

with wavenumber-dependent functions $\phi_{i}, \theta_{j}, \eta_{j}: \mathbf{R} \rightarrow \mathbf{C}$ defined by

$$
\begin{aligned}
\phi_{0}\left(k_{0}\right) & =1, & \phi_{1}\left(k_{0}\right) & =k_{0}, & \phi_{2}\left(k_{0}\right) & =k_{0}^{2}, \\
\phi_{3}\left(k_{0}\right) & =k_{0} \frac{\beta}{\alpha}, & \phi_{4}\left(k_{0}\right) & =k_{0}^{2} \frac{1}{\alpha}, & \phi_{5}\left(k_{0}\right) & =k_{0}^{3} \frac{\gamma}{\alpha}, \\
\theta_{j} & =j k_{0} \eta_{0}, & \eta_{j} & =1, & &
\end{aligned}
$$

and block matrices $\hat{\mathbf{A}}_{i}$ and block vectors $\hat{\boldsymbol{x}}\left(k_{0}\right), \hat{\boldsymbol{b}}_{i}$ given by

$$
\begin{aligned}
\hat{\mathbf{A}}_{i} & =\left[\begin{array}{cc}
\mathbf{A}_{1}^{i} & \mathbf{C}_{12}^{i} \\
\mathbf{C}_{21}^{i} & \mathbf{A}_{2}^{i}
\end{array}\right] \in \mathbf{C}^{\left(N_{1}+N_{2}\right) \times\left(N_{1}+N_{2}\right)}, \\
\hat{\boldsymbol{x}}\left(k_{0}\right) & =\left[\begin{array}{l}
\boldsymbol{x}_{1}\left(k_{0}\right) \\
\boldsymbol{x}_{2}\left(k_{0}\right)
\end{array}\right] \in \mathbf{C}^{\left(N_{1}+N_{2}\right)}, \\
\hat{\boldsymbol{b}}_{i} & =\left[\begin{array}{l}
\boldsymbol{b}_{1}^{i} \\
\boldsymbol{b}_{2}^{i}
\end{array}\right] \in \mathbf{C}^{\left(N_{1}+N_{2}\right)} .
\end{aligned}
$$

A multi-point reduced-order model (ROM) is built from the FE solutions $\boldsymbol{x}\left(k_{0}^{i}\right)$ of Eq. 16 at $M$ expansion points $k_{0}^{1}, \ldots, k_{0}^{M}$, with $M \ll N_{1}+N_{2}$, which are selected adaptively; see Sect. 3.1. The first step is to compute a unitary projection matrix $\mathbf{V} \in \mathbf{C}^{N_{1}+N_{2}, M}$ with

$\operatorname{range}\left(\mathbf{V}_{M}\right)=\operatorname{span}\left\{\boldsymbol{x}\left(k_{0}^{i}\right)\right\}, i=1 \ldots M$.

Then, Galerkin projection leads to a ROM of the form

$$
\begin{aligned}
\left(\sum_{i=1}^{I} \phi_{i}\left(k_{0}\right) \widetilde{\mathbf{A}}_{i}\right) \widetilde{\boldsymbol{x}}\left(k_{0}\right) & =\left(\sum_{j=1}^{J} \theta_{j}\left(k_{0}\right) \widetilde{\boldsymbol{b}}_{j}\right), \\
\widetilde{\boldsymbol{y}}\left(k_{0}\right) & =\left(\sum_{j=1}^{J} \eta_{j}\left(k_{0}\right) \widetilde{\boldsymbol{b}}_{j}\right) \widetilde{\boldsymbol{x}}\left(k_{0}\right)
\end{aligned}
$$

wherein the reduced matrices and vectors are defined as

$$
\begin{aligned}
\widetilde{\mathbf{A}}_{i} & =\mathbf{V}_{M}^{*} \hat{\mathbf{A}}_{i} \mathbf{V}_{M} \in \mathbf{C}^{M \times M}, \\
\widetilde{\boldsymbol{b}}_{i} & =\mathbf{V}_{M}^{*} \hat{\boldsymbol{b}}_{i} \in \mathbf{C}^{M} .
\end{aligned}
$$

Thus, ROM construction requires the solution of the largescale FE-DD system Eq. 16 at each expansion point. Since

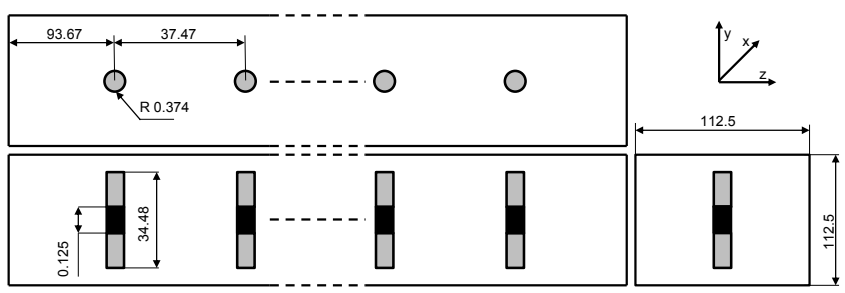

Figure 1. FE model of linear array of dipoles. Black rectangles indicate lumped ports. Dimensions are in $\mathrm{mm}$.

Eq. 16 is solved iteratively, ROM construction time is determined not only by the ROM dimension $M$ but also by the convergence behavior of the linear solver. To improve solver performance, we use an adaptive two-level preconditioner based on the reduced-order system already available at a given adaptive step.

\subsection{Adaptivity}

As demonstrated in Patera and Rozza (2006-2007), the 2norm of the residual $\boldsymbol{r}\left(k_{0}\right)$ of Eq. 16a according to the ROM solution $\boldsymbol{x}\left(k_{0}\right)$,

$$
\begin{aligned}
\boldsymbol{r}\left(k_{0}\right) & =\left(\sum_{j=1}^{J} \theta_{j}\left(k_{0}\right) \hat{\boldsymbol{b}}_{j}\right) \\
& -\left(\sum_{i=1}^{I} \phi_{i}\left(k_{0}\right) \hat{\mathbf{A}}_{i}\right) \mathbf{V}_{M} \boldsymbol{x}\left(k_{0}\right),
\end{aligned}
$$

can be evaluated very efficiently, using reduced-order quantities only. It was suggested in de la Rubia et al. (2009) to employ the relative residual norm $\rho$,

$\rho\left(k_{0}\right)=\frac{\left\|\boldsymbol{r}\left(k_{0}\right)\right\|_{2}}{\left\|\sum_{j=1}^{J} \theta_{j}\left(k_{0}\right) \hat{\boldsymbol{b}}_{j}\right\|_{2}}$,

as an inexpensive error indicator for guiding the placement of the expansion points $k_{0}^{i}$ : At a given stage of ROM generation, $\rho$ is evaluated for a dense sampling $\left\{k_{0}^{S}\right\}$ of the wavenumber interval under investigation, and the following expansion point is chosen where the relative residual is the largest. ROM generation terminates when $\rho$ has fallen below a userdefined threshold $\rho_{0}$ for all $k_{0}^{s}$.

\section{Numerical experiments}

To simplify mathematical analysis and minimize the costs of numerical tests, we consider a structure that is structurally simple and electrically large in a single direction. Specifically, we choose a linear array of $N_{A}$ equally spaced, perfectly conducting dipoles in free space. The geometry of the FE domain is shown in Fig. 1. On the outer surface, absorbing boundary conditions are applied. The dipoles are excited at their centers by lumped ports of impedance $Z_{P}=73 \Omega$. To steer the array pattern towards a given angle $\theta_{s}$, the incident waves at the ports $a_{n}$ are taken of unit magnitude and 


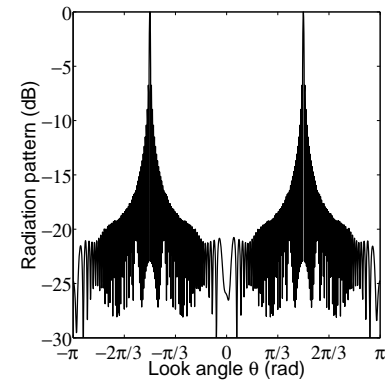

(a) Broadside array; $\phi=0$ rad.

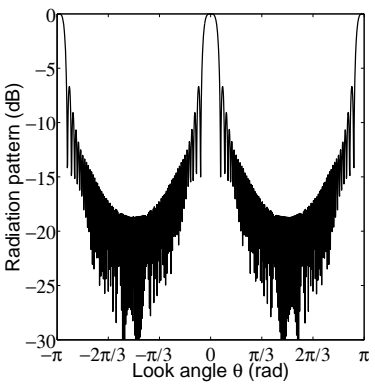

(c) End-fire array; $\phi=0 \mathrm{rad}$.

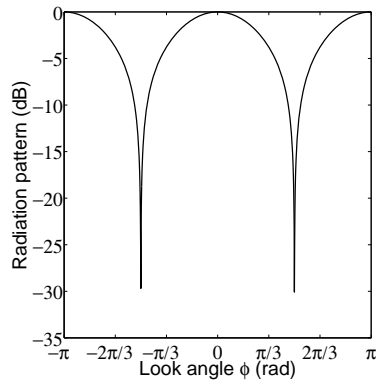

(b) Broadside array; $\theta=\frac{\pi}{2} \mathrm{rad}$.

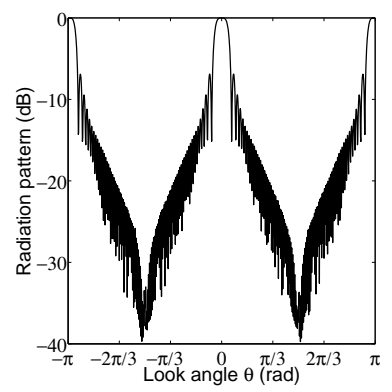

(d) End-fire array; $\phi=\frac{\pi}{2} \mathrm{rad}$.
Figure 2. Normalized radiation patterns of $N_{A}=192$ dipoles at $4 \mathrm{GHz}$, where radiators are one-half wavelength apart.

linear phase distribution,

$a_{n}=e^{-j k_{0} z_{n} \cos \theta_{\mathrm{s}}}$,

where $z_{n}$ is the $z$ coordinate of the dipole axis. Specifically, the limiting cases of broadside $\left(\theta_{\mathrm{S}}=\frac{\pi}{2} \mathrm{rad}\right)$ and endfire $\left(\theta_{\mathrm{s}}=0 \mathrm{rad}\right)$ arrays are considered.

The FE-DD system Eq. (16) is solved by the restarted GM$\operatorname{RES}(30)$ iterative method with stopping criterion $\delta=10^{-6}$; see Saad and Schultz (1986). The ROM termination criterion is $\max \rho\left(k_{0}\right)=10^{-6}$. Preliminary studies on smaller arrays using standard FE techniques rather than DD have confirmed that the behavior of the ROM is mainly determined by the antenna configuration and almost independent of the FE formulation. Thus no additional data are given.

\subsection{Varying number of radiators $N_{A}$}

Let the center frequency $f_{\mathrm{c}}=4 \mathrm{GHz}$ and bandwidth $B=$ $2 \mathrm{GHz}$ be given. We investigate the convergence behavior of the adaptive ROM by varying the number of dipoles in the range $N_{A}=12 \ldots 192$ and, correspondingly, the electric size of the array in the range $(6 \ldots 96) \lambda$ at $4 \mathrm{GHz}$.

Figure 2 presents radiation patterns derived from the ROM, for $N_{A}=192$. The qualitative behavior is as expected: Since the dipoles are one-half wavelength apart at the chosen frequency, $f=4 \mathrm{GHz}$, the radiation patterns of not only the broadside but also the end-fire array are quarter-symmetric.

Figure 3 shows the convergence behavior of the ROM for different numbers of dipoles. The difference between Fig. 3a

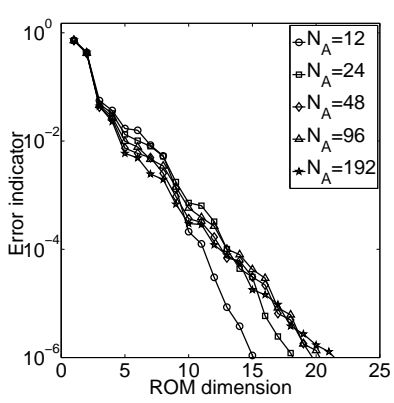

(a) Broadside array.

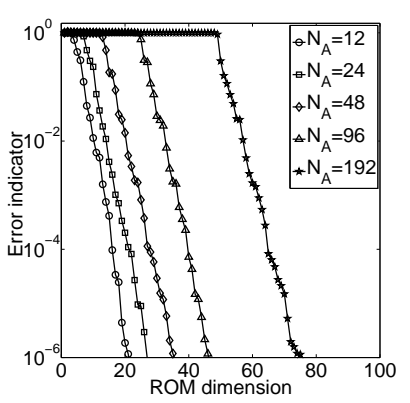

(b) End-fire array.
Figure 3. Error indicator $\max \rho$ vs. ROM dimension for different dipole counts $N_{A}$. Parameters: $f_{\mathrm{c}}=4 \mathrm{GHz}, B=2 \mathrm{GHz}$.

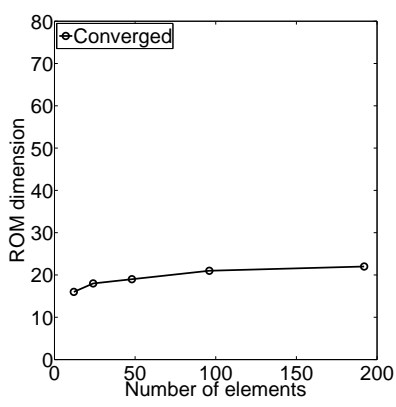

(a) Broadside array.

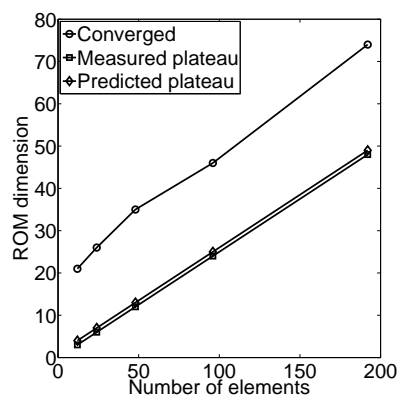

(b) End-fire array.
Figure 4. ROM dimension versus number of dipoles $N_{A}$. Parameters: $f_{\mathrm{c}}=4 \mathrm{GHz}, B=2 \mathrm{GHz}$.

and $\mathrm{b}$ is striking: the broadside array does not exhibit any pre-asymptotic region and leads to exponential convergence rates right from the start whereas the end-fire configuration leads to a pronounced plateau, followed by exponential convergence. In both cases, convergence rates in the asymptotic region deteriorate slowly with increasing number of dipoles. In consequence, ROM dimension remains almost constant for the broadside array, as illustrated by Fig. 4a. In the endfire case, in contrast, both plateau width and ROM dimension increase linearly with the number of dipoles; see Fig. 4b. For completeness, the dimensions of the underlying FE-DD systems and corresponding ROMs for different numbers of dipoles are collected in Table 1.

\subsection{Varying bandwidth $B$}

For given center frequency $f_{\mathrm{c}}=4 \mathrm{GHz}$ and number of dipoles $N_{A}=48$, we construct ROMs of varying bandwidth $B=(0.5 \ldots 4) \mathrm{GHz}$.

Figure 5 illustrates the frequency response of the active reflection coefficient for one dipole at the center and one at the end of the array. It can be seen that broadside and end-fire arrays behave similarly: in both cases, the detuning effects of the periodic environment on the center dipole are clearly visible. 
Table 1. Computational data for $f_{\mathrm{c}}=4 \mathrm{GHz}, B=2 \mathrm{GHz}$.

\begin{tabular}{lrrr}
\hline Number of & \multicolumn{3}{c}{ Dimension } \\
Radiators & FE-DD & \multicolumn{2}{c}{ ROM } \\
& - & End-fire & Broadside \\
\hline 12 & $1.8 \cdot 10^{6}$ & 21 & 16 \\
24 & $3.2 \cdot 10^{6}$ & 27 & 18 \\
48 & $5.9 \cdot 10^{6}$ & 35 & 19 \\
96 & $11.4 \cdot 10^{6}$ & 46 & 21 \\
192 & $22.5 \cdot 10^{6}$ & 75 & 22 \\
\hline
\end{tabular}

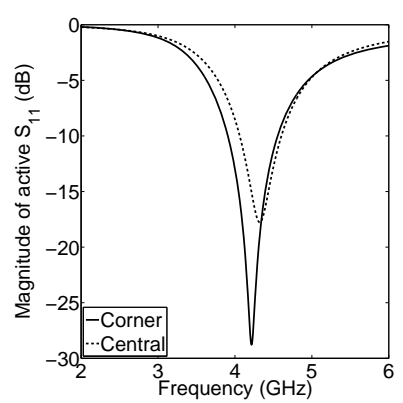

(a) Broadside: magnitude.

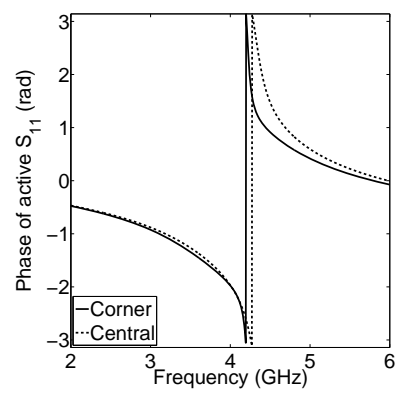

(c) Broadside: phase.

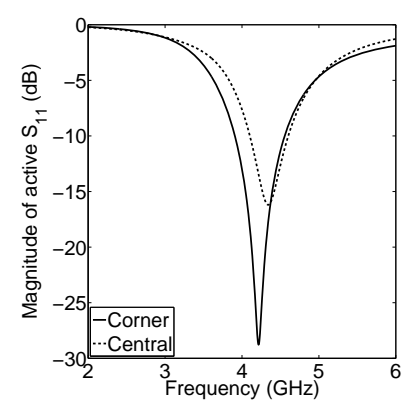

(b) End-fire: magnitude.

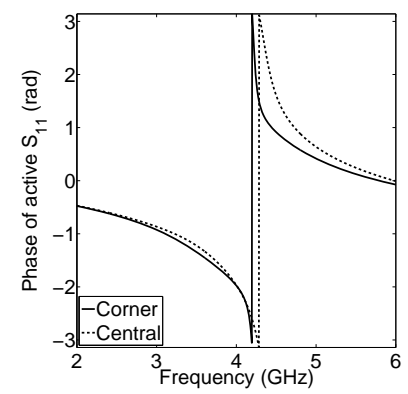

(d) End-fire: phase.
Figure 5. Frequency response of active reflection coefficient for selected dipoles. Array size: $N_{A}=48$ elements.

Figure 6 presents the convergence behavior of the adaptive ROM for different choices of bandwidth: the broadband array leads to a very short pre-asymptotic region at most, whereas the end-fire configuration exhibits a pronounced plateau. In the asymptotic region, both cases result in exponential convergence which deteriorates with increasing bandwidth. Figure 7 illustrates the behavior of the ROM dimension as a function of bandwidth for both broadside and end-fire configurations.

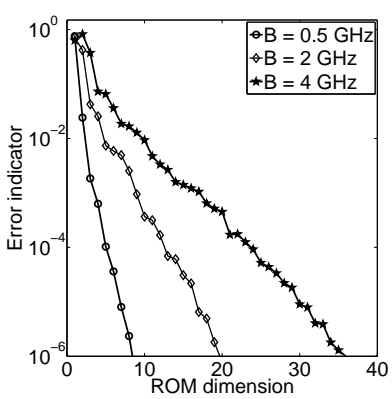

(a) Broadside array.

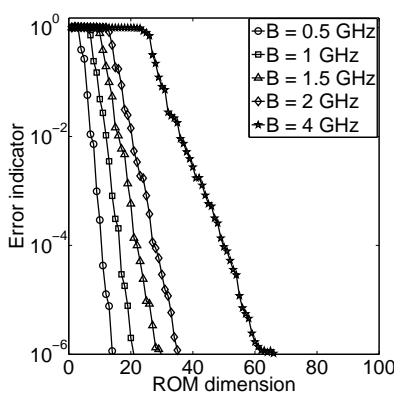

(b) End-fire array.
Figure 6. Error indicator max $\rho$ versus ROM dimension for different bandwidths $B$. Parameters: $N_{A}=48, f_{\mathrm{c}}=4 \mathrm{GHz}$.

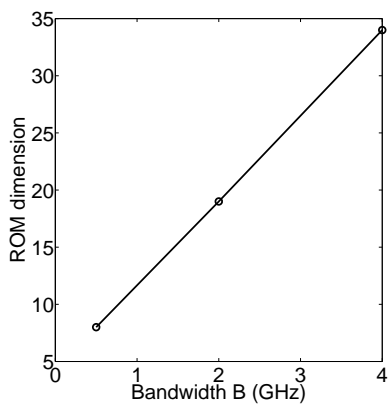

(a) Broadside array.

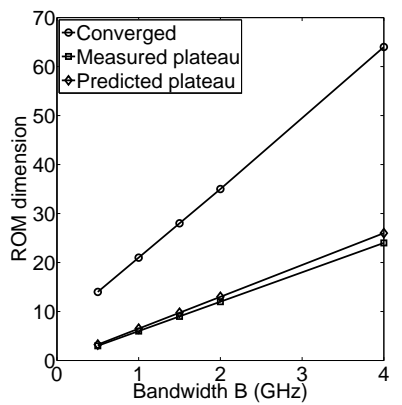

(b) End-fire array.
Figure 7. ROM dimension versus bandwidth $B$. Parameters: $N_{A}=$ $48, f_{\mathrm{c}}=4 \mathrm{GHz}$.

\section{Proposed explanation of convergence properties}

A first hint at the origin of the peculiar convergence behavior is given by the distributions of the electric near-fields on the side face of the FE domain plotted in Fig. 8: Fig. 8a and b illustrate that, for the broadside array, the field distribution does not change much over the frequency range $(2-4) \mathrm{GHz}$, because the excitations are in phase, and the domain is electrically small perpendicular to the $z$ direction. In case of the end-fire configuration, however, the fields vary strongly with frequency, in accordance with Eq. (27); see Fig. 8c and d.

Changes in spatial distribution are crucial because the projection-based MOR of Sect. 3 is an approximation method that employs the electric field at the expansion wavenumbers, corresponding to the FE solutions $\boldsymbol{x}\left(k_{0}^{i}\right)$, as basis functions in the spatial domain.

As long as the structure is electrically large in the $z$ direction only, and provided that the electric behavior of a single radiator does not change fundamentally over the considered frequency range, one may decompose the fields into localized contributions that do not vary much with frequency and wave-like components $w\left(z, k_{0}\right)$ in $z$ direction, in accordance with the steering angle $\theta_{\mathrm{s}}$ :

$w\left(z, k_{0}\right)=e^{-j k_{0} z \cos \theta_{\mathrm{s}}}=e^{-j 2 \pi f \frac{z}{c_{0}} \cos \theta_{\mathrm{s}}}$. 
Herein, $c_{0}$ is the vacuum speed of light. It is therefore a necessary requirement for convergence of the ROM that the projection space of Eq. (21) must resolve all $w\left(z, k_{0}\right)$ that get excited by Eq. (27) over the considered frequency range. The authors suppose that it is the non-fulfillment of such condition that causes the plateau in the convergence behavior. Our mathematical model is as follows: we express the frequency as

$f=f_{\mathrm{c}}+\Delta f$

with center frequency $f_{\mathrm{c}}$ and the equivalent baseband frequency $\Delta f$. By introducing the spatial frequency

$f_{\mathrm{z}}=\Delta f \frac{\cos \theta_{\mathrm{s}}}{c_{0}}$,

the waves (Eq. 28) take the form

$w\left(z, f_{\mathrm{Z}}\right)=e^{-j 2 \pi f_{\mathrm{c}} \frac{z}{c_{0}} \cos \theta_{\mathrm{s}}} e^{-j 2 \pi f_{\mathrm{z}} z}$

with

$f_{\mathrm{Z}} \in\left[-\frac{B \cos \theta_{\mathrm{s}}}{2 c_{0}}, \frac{B \cos \theta_{\mathrm{s}}}{2 c_{0}}\right]$.

We next assume an equidistant spatial sampling in $z$ direction and apply the discrete Fourier transformation. According to Oppenheim and Schafer (1989, p. 698), the length $L$ of the field domain leads to a spatial frequency resolution of

$\delta f_{\mathrm{z}}=\frac{1}{L}$.

Thus, the minimum number of frequencies $M_{\text {min }}$ required to sample the interval (Eq. 32) is given by

$M_{\min }=\frac{B L \cos \theta_{\mathrm{s}}}{c_{0}}$.

\subsection{Varying number of radiators}

Equation (34) suggests that the required number of expansion frequencies $M_{\min }$ depends on the length $L$ of the computational domain weighted by the cosine of the steering angle, $L \cos \theta_{\mathrm{s}}$, rather than the number of radiators. However, it is common use to embed the array in an air buffer of constant size: In the examples of Sect. $4.1, L$ was always taken as

$L=\left(N_{A}+4\right) d$,

where $d$ is the distance between radiators. Hence

$M_{\text {min }}=\frac{B\left(N_{A}+4\right) d \cos \theta_{\mathrm{s}}}{c_{0}}$.

Equation 36 predicts that plateaus do not occur in the case of broadside arrays, $\cos \theta_{\mathrm{s}}=0$, whatever the number of radiators $N_{a}$. This agrees with the experimental findings of Fig. 3a. For the end-fire case, $\cos \theta_{\mathrm{s}}=1$, the results of Eq. (36) have been included in Fig. 6a. It can be seen that the theoretical results are slightly conservative. However, they track the actual width of the plateau very well, over the entire range of 12-192 dipoles.

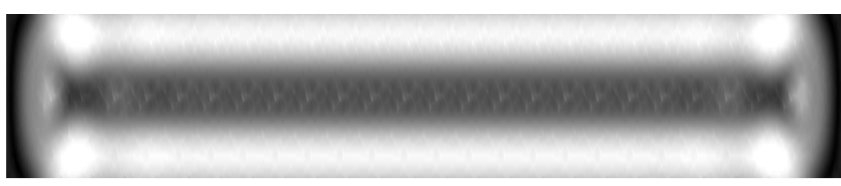

(a) Broadside array at $2 \mathrm{GHz}, \theta_{s}=\frac{\pi}{2}$.

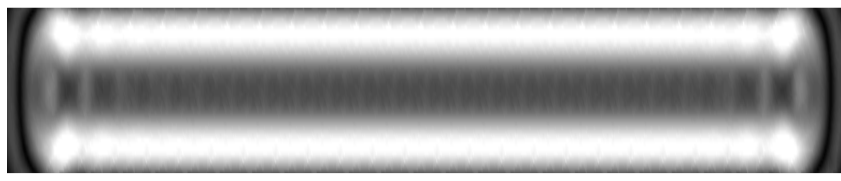

(b) Broadside array at $4 \mathrm{GHz}, \theta_{s}=\frac{\pi}{2}$.

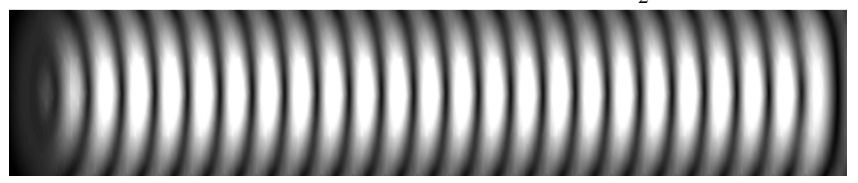

(c) Endfire array at $2 \mathrm{GHz}, \theta_{s}=0$.

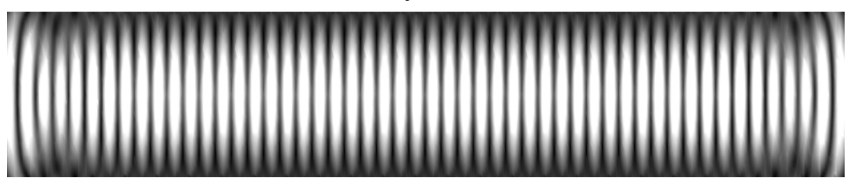

(d) Endfire array at $4 \mathrm{GHz}, \theta_{s}=0$.

Figure 8. Magnitude of electric field on outer boundary of FE domain. Array size: $N_{A}=48$ dipoles.

\subsection{Varying bandwidth}

Again, Eq. (36) predicts no plateau for broadside arrays, which agrees well with the experimental findings of Fig. 6 a. The authors conjecture that the short pre-asymptotic region of 2 iterations present in Fig. 6 a for $B=4 \mathrm{GHz}$ is caused by the fact that, at the highest operating frequency, the transversal dimensions of the FE model correspond to $2.25 \lambda$ which is no longer electrically small. For the the end-fire case, $\cos \theta_{\mathrm{s}}=1$, the theoretical results of Eq. (36) have been included in Fig. 7b. Again, they are somewhat conservative but track the actual width of the plateau very well, for all considered bandwidths.

\section{Further validations}

\subsection{Effectiveness of equidistant expansion frequencies}

The proposed model predicts that adaptive MOR construction based on some error indicator is not necessary in the pre-asymptotic region. Instead, it will suffice to take $M_{\min }$ frequencies spaced equally over $B$. 


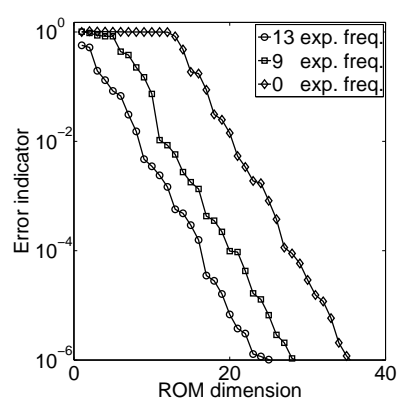

(a) Adaptive part of ROM.

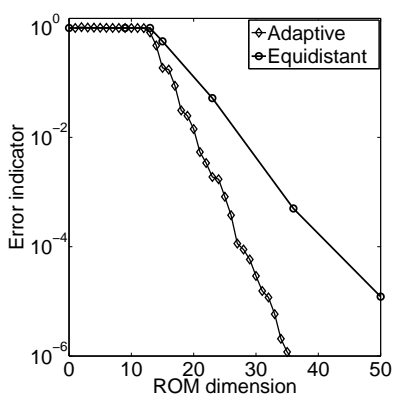

(b) Adaptive versus equidistant expansion frequencies.

Figure 9. End-fire array $\left(\theta_{\mathrm{s}}=0\right)$ of $N_{A}=48$ dipoles, with $f_{\mathrm{c}}=$ $4 \mathrm{GHz}$ and $B=2 \mathrm{GHz}$ : Error indicator $\rho\left(k_{0}\right)$ versus ROM dimension. Parameter: number of pre-computed equidistant expansion frequencies.

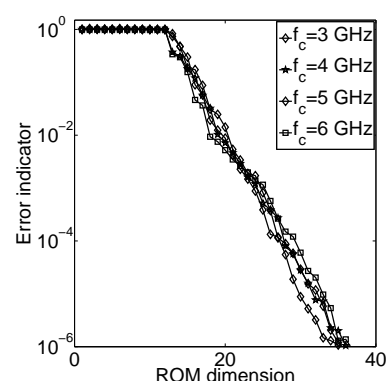

(a) ROM error indicator $\max \rho$ versus ROM dimension.

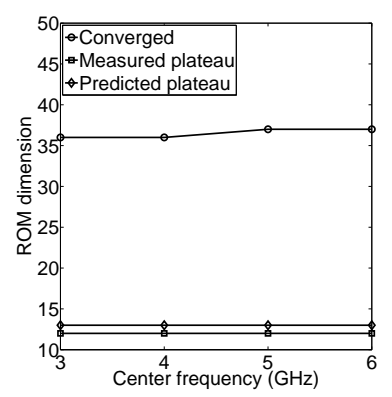

(b) ROM dimension versus center frequency.

Figure 10. End-fire array $\left(\theta_{\mathrm{S}}=0\right)$ of $N_{A}=48$ dipoles: dependence of adaptive ROM on center frequency $f_{\mathrm{c}}$ for constant bandwidth of $B=2 \mathrm{GHz}$.

To test this hypothesis, we consider an end-fire array of 48 dipoles, center frequency $4 \mathrm{GHz}$, and bandwidth $4 \mathrm{GHz}$. According to Eq. (36), 13 equidistant expansion frequencies are required to prevent a plateau from occurring. We initialize the ROM basis with the solutions at $n_{\text {eq }}$ equidistant frequency points before starting the adaptive MOR process. Figure 9a shows convergence curves for different choices of $n_{\text {eq }}$. For $n_{\text {eq }}<M_{\min }$, a plateau occurs, and its width is approximately $N_{\min }-n_{\text {eq }}$. At $n_{\text {eq }}=M_{\min }$, the onset of exponential convergence is immediate, which confirms that the corresponding ROM space contains all critical information.

To demonstrate the advantages of adaptive MOR in the asymptotic region, Fig. $9 \mathrm{~b}$ gives a comparison to a series of ROMs that always use equidistant expansion points: the convergence rate of the adaptive method is significantly higher.

\subsection{Varying center frequency}

The proposed model also implies that the width of the plateau will be independent of the center frequency $f_{\mathrm{c}}$.

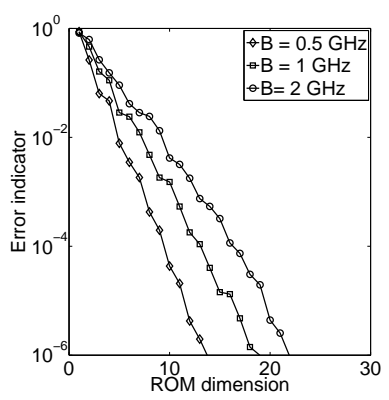

(a) ROM error indicator $\max \rho$ versus ROM dimension.

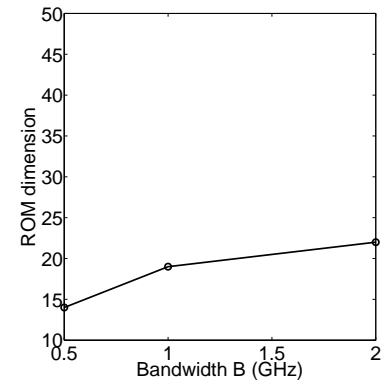

(b) ROM dimension versus bandwidth.
Figure 11. Dependence of adaptive ROM on bandwidth $B$ for single excited radiator at center of 48 dipoles; $f_{\mathrm{c}}=4 \mathrm{GHz}$.

In our numerical test, we investigate an end-fire array of 48 dipoles and bandwidth $2 \mathrm{GHz}$. Equation 36 predicts a constant plateau width of 13 iterations. Figure 10a gives convergence curves for different center frequencies in the range 3-6 GHz, and Fig. 10a presents the corresponding ROM dimensions. It can be seen that the plateau width is constant, at 12 iterations.

\subsection{Spatial distribution of excitations}

Whenever the levels of sidelobes are of importance, excitations of tapered amplitude are employed. In the examples above, however, it was assumed that the excitations of the dipoles were of constant magnitude and linear phase shift. The authors believe that, despite its simplicity, this wave form is well chosen because it strongly excites wave-like fields along the $z$ direction and allows to vary the spatial periodicity in the $z$ direction from infinity to the actual wavelength at the highest operating frequency; see Eq. (28).

To complement our numerical tests, we investigate the frequency response as a function of bandwidth for a spatially impulse-like excitation, a single driven dipole at the center of an array of 48 dipoles, with $f_{\mathrm{c}}=4 \mathrm{GHz}$. Figure 11 illustrates the convergence behavior for different bandwidths in the range $0.5-2 \mathrm{GHz}$ : there are no plateaus, and iteration counts are moderate. The nearfield plots of Fig. 12 suggest the following qualitative explanation: the dipoles adjacent to the driven element direct the fields in the $z$ direction. However, since their ports provide nearly matched terminations, they also absorb a great amount of energy. In consequence, the fields are strongly damped, so that the amplitudes of many wave-like modes along the $z$ direction become very weak. Since the MOR process is driven by the system response, such modes are disregarded. 


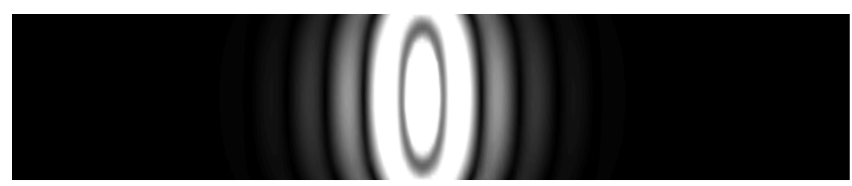

(a) Frequency: $2 \mathrm{GHz}$.

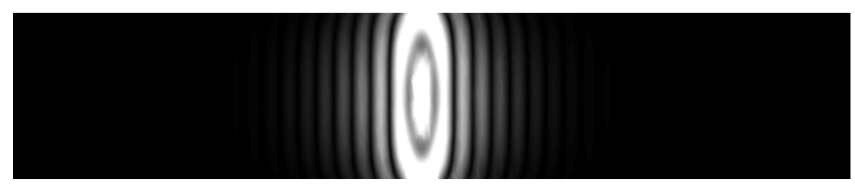

(b) Frequency: $4 \mathrm{GHz}$.

Figure 12. Magnitude of electric field on boundary of FE domain for single excited radiator at center of 48 dipoles.

\section{Conclusions and outlook}

The numerical studies presented in this paper have shown that projection-based MOR converges even for linear arrays of large electrical size ( $144 \lambda$ at $6 \mathrm{GHz})$. Depending on excitation, the convergence curves may exhibit a pronounced preasymptotic region which depends on steering angle, electric length of the model domain, and bandwidth; the worst case occurs in the end-fire direction, $\left|\theta_{\mathrm{s}}\right|=\frac{\pi}{2} \mathrm{rad}$.

The proposed mathematical model has proved to predict the width of the plateau very well. It also allows to replace the adaptive, error-driven selection of MOR expansion frequencies, which is intrinsically sequential, in the pre-asymptotic region by a well-defined number of equally spaced points. Not only does this remove the computational burden of error estimation; it is also perfectly suited for parallelization. Note that, in practice, the impact of the plateau on overall performance may be much higher than in the examples given in this paper: The MOR termination criterion of $10^{-6}$ was chosen very low to demonstrate numerical robustness and lack of stagnation. In many real-world applications, residual norms in the order of $10^{-2} \ldots 10^{-3}$ will suffice. Thus the asymptotic region will be much shorter whereas the plateau width will stay the same.

In the case of planar arrays, the authors conjecture from Eq. (34) that the plateau width will be in the order of $\left(B L\left|\cos \theta_{\mathrm{s}}\right| / c_{0}\right)^{2}$. More detailed studies are the subject of ongoing research.

Edited by: R. Schuhmann

Reviewed by: two anonymous referees

\section{References}

Buffa, A. and Ciarlet, P.: On traces for functional spaces related to Maxwell's equations Part I: An integration by parts formula in Lipschitz polyhedra, Mathemat. Method. Appl. Sci., 24, 9-30, 2001.

Chen, Y., Hesthaven, J., Maday, Y., and Rodríguez, J.: Certified Reduced Basis Methods and Output Bounds for the Harmonic Maxwell's Equations, SIAM J. Scientif. Comput., 32, 970-996, 2010.

de la Rubia, V., Razafison, U., and Maday, Y.: Reliable Fast Frequency Sweep for Microwave Devices via the Reduced-Basis Method, IEEE Transactions on Microwave Theory and Techniques, 57, 2923-2937, 2009.

Dolean, V., Gander, M. J., Lanteri, S., Lee, J.-F., and Peng, Z.: Effective transmission conditions for domain decomposition methods applied to the time-harmonic curl-curl Maxwell's equations, J. Computat. Phys., 280, 232-47, 2015.

Hesthaven, J. S., Stamm, B., and Zhang, S.: Certified Reduced Basis Method for the Electric Field Integral Equation, SIAM J. Scientif. Comput., 34, A1777-A1799, 2012.

Lee, S.-C., Vouvakis, M. N., and Lee, J.-F.: A non-overlapping domain decomposition method with non-matching grids for modeling large finite antenna arrays, J. Computat. Phys., 203, 1-21, 2005.

Oppenheim, A. and Schafer, R.: Discrete-Time Signal Processing, Prentice Hall, international Edn., 1989.

Patera, A. T. and Rozza, G.: Reduced Basis Approximation and A Posteriori Error Estimation for Parametrized Partial Differential Equations, to appear in (tentative rubric) MIT Pappalardo Graduate Monographs in Mechanical Engineering, Version 1.0, 20062007.

Rawat, V. and Lee, J.: Nonoverlapping Domain Decomposition with Second Order Transmission Condition for the Time-Harmonic Maxwell's Equations, SIAM J. Scient. Comput., 32, 3584-3603, 2010.

Rawat, V. and Lee, J.-F.: Treatment of cement variables in the domain decomposition method for Maxwell's equations, in: Antennas and Propagation Society International Symposium, 2007 IEEE, 5937-5940, 2007.

Saad, Y. and Schultz, M.: GMRES: A Generalized Minimal Residual Algorithm for Solving Nonsymmetric Linear Systems, SIAM J. Scient. Stat. Comput., 7, 856-869, 1986. 